\title{
Reduction to dimension two of local spectrum for $A H$ algebra with ideal property
}

\author{
Chunlan Jiang
}

\begin{abstract}
A $C^{*}$-algebra $A$ has ideal property if any ideal $I$ of $A$ is generated as a closed two sided ideal by the projections inside the ideal. Suppose that the limit $C^{*}$-algebra $A$ of inductive limit of direct sums of matrix algebras over spaces with uniformly bounded dimension has ideal property. In this paper we will prove that $A$ can be written as an inductive limit of certain very special subhomogeneous algebras, namely, direct sum of dimension drop interval algebras and matrix algebras over 2-dimensional spaces with torsion $H^{2}$ groups.
\end{abstract}

\section{§1. Introduction}

An $A H$ algebra is a nuclear $C^{*}$-algebra of the form $A=\underset{\lim }{\longrightarrow}\left(A_{n}, \phi_{n, m}\right)$ with $A_{n}=$ $\bigoplus_{i=1}^{t_{n}} P_{n, i} M_{[n, i]}\left(C\left(X_{n, i}\right)\right) P_{n, i}$, where $X_{n, i}$ are compact metric spaces, $t_{n},[n, i]$ are positive integers, $M_{[n, i]}\left(C\left(X_{n, i}\right)\right)$ are algebras of $[n, i] \times[n, i]$ matrices with entries in $C\left(X_{n, i}\right)$ - the algebra of complex - valued functions on $X_{n, i}$, and finally $P_{n, i} \in M_{[n, i]}\left(C\left(X_{n, i}\right)\right)$ are projections (see [Bla]). If we further assume that $\sup _{n, i} \operatorname{dim}\left(X_{n, i}\right)<+\infty$ and $A$ has ideal property - each ideal $I$ of $A$ is generated by the projections inside the ideal, then it is proved in [GJLP1-2] that $A$ can be written as inductive limit of $B_{n}=\bigoplus_{i=1}^{s_{n}} P_{n, i}^{\prime} M_{[n, i]}\left(C\left(Y_{n, i}\right)\right) P_{n, i}^{\prime}$.

In this paper, we will further reduce the dimension of local spectra (that is the spectra of $A_{n}$ or $B_{n}$ above) to 2 (instead of 3 ). Namely, the above $A$ can be written as inductive limit of direct sum of matrix algebras over the $\{p t\},[0,1], S^{1}, T_{I I, k}\left(\right.$ no $T_{I I I, k}$ and $\left.S^{2}\right)$ and $M_{l}\left(I_{k}\right)$, where $I_{k}$ is dimension drop interval algebra,

$$
I_{k}=\left\{f \in C\left([0,1], M_{k}(\mathbb{C})\right), f(0)=\lambda \mathbf{1}_{k}, f(1)=\mu \mathbf{1}_{k}, \lambda, \mu \in \mathbb{C}\right\} .
$$

In this paper, we will also call $\bigoplus_{i=1}^{s} M_{l_{i}}\left(I_{k_{i}}\right)$ a dimension drop-algebra.

This result unifies the theorems of [DG] and [EGS] (for the rank zero case) and [Li4] (for the simple case). Note that $\mathrm{Li}^{\prime} \mathrm{s}$ reduction theorem was not used in the classification of simple $A H$ algebra, and $\mathrm{Li}^{\prime}$ s proof depends on the classification of simple $A H$ algebra (see [Li4] and [EGL]). For our case, the reduction theorem is an important step toward the classification (see [GJL]). The proof is more difficult than $\mathrm{Li}^{\prime}$ s case. For example, in the case of $A H$ 
algebra with ideal property, one can not remove the space $S^{2}$ without introduce $M_{l}\left(I_{k}\right)$ (for the simple case, the space $S^{2}$ is removed from the list of spaces in [EGL] without introduced dimension drop algebras). Another point is that, in the simple $A H$ algebras, one can assume each partial map $\phi_{n, m}^{i, j}$ is injective, but in $A H$ algebras with ideal property, we can not make such assumption. For the classification of real rank zero $A H$ algebras, we refer to the readers [Ell1], [EG1-2], [D1-2], [G1-4] and [DG]. For the classification simple $A H$ algebra, we refer to the readers [Ell2-3], [Li1-3], [G5] and [EGL1-2].

The paper is organized as follows.

In section 2, we will do some necessary preparation. In section 3, we will prove our main theorem.

\section{§2. Preparation}

We will adopt all the notation from section 2 of [GJLP2]. For example we refer the reader to [GJLP2] for the concepts of $G-\delta$ multiplicative maps (see definition 2.2 there), spectral variation $S P V(\phi)$ of a homomorphism $\phi$ (see 2.12 there) weak variation $\omega(F)$ of a finite set $F \subset Q M_{N}(C(X)) Q$ (see 2.16 there).

As in 2.17 of [GJLP2], we shall use $\bullet$ to denote any possible integer.

2.1. In this article, without lose of generality we will assume the $A H$ algebras $A$ are inductive limit of

$$
A=\lim \left(A_{n}=\bigoplus_{i=1}^{t_{n}} M_{[n, i]}\left(C\left(X_{n, i}\right)\right), \phi_{n, m}\right),
$$

where $X_{n, i}$ are the spaces of $\{p t\},[0,1], S^{1}, T_{I I, k}, T_{I I I, k}$, and $S^{2}$. (Note by the main theorem of [GJLP2], all $A H$ algebras with ideal property and with no dimension growth are corner subalgebras of the above form (also see 2.7 of [GJLP2]).)

2.2. Recall a projection $P \in M_{k}(C(X))$ is called a trivial projection if it is unitarily equivalent to $\left(\begin{array}{cc}\mathbf{1}_{k_{1}} & 0 \\ 0 & 0\end{array}\right)$ for $k_{1}=\operatorname{rank}(P)$. If $P$ is a trival projection and $\operatorname{rank}(P)=k_{1}$, then

$$
P M_{k}(C(X)) P \cong M_{k_{1}}(C(X))
$$

2.3. Let $X$ be a connected finite simplicial complex, $A=M_{k}(C(X))$, a unital $*$ homomorphism $\phi: A \longrightarrow M_{l}(A)$ is called a (unital) simple embedding if it is homotopic to the homomorphism $i d \oplus \lambda$, when $\lambda: A \longrightarrow M_{l-1}(A)$ is defined by

$$
\lambda(f)=\operatorname{diag}(\underbrace{f\left(x_{0}\right), f\left(x_{0}\right), \cdots, f\left(x_{0}\right)}_{l-1}),
$$

for a fixed base point $x_{0} \in X$. 
The following two lemmas are special cases of Lemma 2.15 of [EGS] (also see 2.12 of [EGS]).

Lemma 2.4. (c.f 2.12 or case 2 of 2.15 in [EGS]). For any finite set $F \subset A=M_{n}\left(C\left(T_{I I I, k}\right)\right)$ and $\varepsilon>0$, there is a unital simple embedding $\phi: A \longrightarrow M_{l}(A)$ (for $l$ large enough) and a $C^{*}$-algebra $B \subset A$, which is a direct sum of dimension drop algebras and a finite dimensional $C^{*}$-algebra such that

$$
\operatorname{dist}(\phi(f), B)<\varepsilon, \quad \forall f \in F .
$$

Lemma 2.5.(see case 1 of 2.15 in [EGS]) For any finite set $F \subset M_{n}\left(C\left(S^{2}\right)\right)$ and $\varepsilon>0$, there is a unital simple embedding $\phi: A \longrightarrow M_{l}(A)$ (for $l$ large enough) and a $C^{*}$-algebra $B \subset A$, which is a finite dimensional $C^{*}$-algebra such that

$$
\operatorname{dist}(\phi(f), B)<\varepsilon, \quad \forall f \in F .
$$

The following lemma is well known:

Lemma 2.6.(see $[\mathrm{G} 5,4.40])$ For any $C^{*}$-algebra $A$ and finite set $F \subset A, \varepsilon>0$, there is a finite set $G \subset A$ and $\eta>0$ such that if $\phi: A \longrightarrow B$ is a homomorphism and $\psi: A \longrightarrow B$ is a completely positive linear map, satisfing the following

$$
\|\phi(g)-\psi(g)\|<\eta, \quad \forall g \in G,
$$

then $\psi$ is the $F-\varepsilon$ multiplicative.

Lemma 2.7. Let $A=M_{n}\left(C\left(T_{I I I, k}\right)\right)$ or $M_{n}\left(C\left(S^{2}\right)\right)$. And let a finite set $F \subset A$ and $\varepsilon>0$, there is a diagram

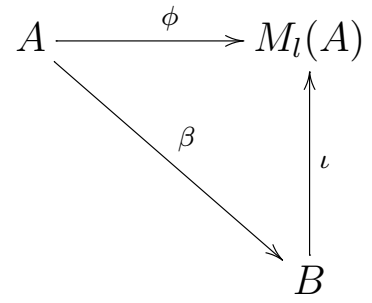

with the following conditions.

(1) $\phi$ is a simple embedding,

(2) if $A=M_{n}\left(C\left(S^{2}\right)\right)$, then $B$ is a finite dimensional $C^{*}$-algebra, and if $A=M_{n}\left(C\left(T_{I I I, k}\right)\right)$, then $B$ is a direct sum of dimension drop $C^{*}$-algebras and a finite dimensional $C^{*}$-algebra, and $\iota$ is an inclusion,

(3) $\|\iota \circ \beta(f)-\phi(f)\|<\varepsilon, \forall f \in F$, and $\beta$ is $F-\varepsilon$ multiplicative.

Proof. Let $G$ and $\eta$ be as Lemma 2.6 for $F$ and $\varepsilon$. Apply Lemma 2.4 or Lemma 2.5 to $A$, $F \cup G \subset A$ and $\frac{1}{3} \min (\varepsilon, \eta)$. One can find a unital simple embedding $\phi: A \longrightarrow M_{l}(A)$, and 
an sub- $C^{*}$-algebra $B \subset M_{l}(A)$ as required in condition (2) such that

$$
\operatorname{dist}(\phi(f), B)<\frac{1}{3} \min (\varepsilon, \eta), \quad \forall f \in F .
$$

Choose a finite $\widetilde{F} \subset B$ such that

$$
\operatorname{dist}(\phi(f), \widetilde{F})<\frac{1}{3} \min (\varepsilon, \eta), \quad \forall f \in F .
$$

Since $B$ is a nuclear $C^{*}$-algebra, there are two completely positive linear maps

$$
\lambda_{1}: B \longrightarrow M_{N}(\mathbb{C}) \text { and } \lambda_{2}: M_{N}(\mathbb{C}) \longrightarrow B
$$

such that

$$
\left\|\lambda_{2} \circ \lambda_{1}(g)-g\right\|<\frac{1}{3} \min (\varepsilon, \eta), \quad \forall g \in \widetilde{F}
$$

Using Arveson extension theorem, one can extend $\lambda_{1}: B \longrightarrow M_{N}(\mathbb{C})$ to a map $\beta_{1}: M_{l}(A) \longrightarrow M_{N}(\mathbb{C})$. Then it is a straight forward to prove that

$$
\beta=\lambda_{2} \circ \beta_{1} \circ \phi: A \longrightarrow B
$$

is as desired.

The following is modification of Theorem 3.8 of [GJLP2].

Proposition 2.8. Let $\lim _{n \rightarrow \infty}\left(A_{n}=\bigoplus_{i=1}^{t_{n}} M_{[n, i]}\left(C\left(X_{n, i}\right)\right), \phi_{n, m}\right)$ be $A H$ inductive limit with ideal property, with $X_{n, i}$ being one of $\{p t\},[0,1], S^{1}, T_{I I, k}, T_{I I I, k}$, or $S^{2}$. Let $B=\bigoplus_{i=1}^{s} B^{i}$, where $B^{i}=M_{l_{i}}\left(C\left(Y_{i}\right)\right)$, with $Y_{i}$ being $\{p t\},[0,1], S^{1}$, or $T_{I I, k}$, (no $T_{I I I, k}$ or $\left.S^{2}\right)$ or $B^{i}=$ $M_{l_{i}}\left(I_{k_{i}}\right)$-dimension drop $C^{*}$-algebras. Suppose that

$$
\widetilde{G}\left(=\oplus \widetilde{G}^{i}\right) \subset G\left(=\oplus G^{i}\right) \subset B\left(=\oplus B^{i}\right),
$$

is a finite set, $\varepsilon_{1}$ is a positive number with $\omega\left(\widetilde{G}^{i}\right)<\varepsilon_{1}$, if $Y_{i}=T_{I I, k}$, and $L$ is any positive integer. Let $\alpha: B \longrightarrow A_{n}$ be any homomorphism. Denote

$$
\alpha\left(\mathbf{1}_{B}\right):=R\left(=\oplus R^{i}\right) \in A_{n}\left(=\oplus A_{n}^{i}\right) .
$$

Let $F \subset R A_{n} R$ be any finite set and $\varepsilon<\varepsilon_{1}$ be any positive number. It follows that there are $A_{m}$, and mutually orthogonal projections $Q_{0}, Q_{1}, Q_{2} \in A_{m}$ with

$$
\phi_{n, m}(R)=Q_{0}+Q_{1}+Q_{2}
$$

a unital map $\theta_{0} \in \operatorname{Map}\left(R A_{n} R, Q_{0} A_{m} Q_{0}\right)_{1}$, two unital homomorphisms $\theta_{1} \in \operatorname{Hom}\left(R A_{n} R, Q_{1} A_{m} Q_{1}\right)_{1}$, $\xi \in \operatorname{Hom}\left(R A_{n} R, Q_{2} A_{m} Q_{2}\right)_{1}$ such that

(1) $\left\|\phi_{n, m}(f)-\left(\theta_{0}(f) \oplus \theta_{1}(f) \oplus \xi(f)\right)\right\|<\varepsilon$, for all $f \in F$,

(2) there is a unital homomorphism

$$
\alpha_{1}: B \longrightarrow\left(Q_{0}+Q_{1}\right) A_{m}\left(Q_{0}+Q_{1}\right)
$$


such that

$$
\left\|\alpha_{1}(g)-\left(\theta_{0}+\theta_{1}\right) \circ \alpha(g)\right\|<3 \varepsilon_{1} \quad \forall g \in \widetilde{G}_{i}, \quad \text { if } B^{i} \text { is of form } \quad M_{\bullet}\left(T_{I I, k}\right)
$$

and

$$
\left\|\alpha_{1}(g)-\left(\theta_{0}+\theta_{1}\right) \circ \alpha(g)\right\|<\varepsilon, \quad \forall g \in G^{i}, \quad \text { if } B^{i} \text { is not of form } M_{\bullet}\left(T_{I I, k}\right) .
$$

(3) $\theta_{0}$ is $F-\varepsilon$ multiplicative and $\theta_{1}$ satisfies that

$$
\theta_{1}^{i, j}([e]) \geqslant L \cdot\left[\theta_{0}^{i, j}\left(R^{i}\right)\right] .
$$

(4) $\xi$ factors through a $C^{*}$-algebra $\mathrm{C}$ - a direct sum of matrix algebras over $\mathrm{C}[0,1]$ as

$$
\xi: R A_{n} R \stackrel{\xi_{1}}{\rightarrow} C \stackrel{\xi_{2}}{\rightarrow} Q_{2} A_{m} Q_{2} .
$$

Proposition 2.9. Let $\lim _{n \rightarrow \infty}\left(A_{n}=\bigoplus_{i=1}^{t_{n}} M_{[n, i]}\left(C\left(X_{n, i}\right)\right), \phi_{n, m}\right)$ be $A H$ inductive limit with ideal property, with $X_{n, i}$ being one of $\{p t\},[0,1], S^{1}, T_{I I, k}, T_{I I I, k}$ or $S^{2}$. Let $B=\bigoplus_{i=1}^{s} B^{i}$, where $B^{i}=M_{l_{i}}\left(C\left(Y_{i}\right)\right)$, with $Y_{i}$ being $\{p t\},[0,1], S^{1}$ or $T_{I I, k}$, (no $T_{I I I, k}$ or $S^{2}$ ) or $B^{i}=$ $M_{l_{i}}\left(I_{k_{i}}\right)$-dimension drop $C^{*}$-algebras. Suppose that

$$
\widetilde{G}\left(=\oplus \widetilde{G}^{i}\right) \subset G\left(=\oplus G^{i}\right) \subset B\left(=\oplus B^{i}\right),
$$

is a finite set, $\varepsilon_{1}$ is a positive number with $\omega\left(\widetilde{G}^{i}\right)<\varepsilon_{1}$, if $Y_{i}=T_{I I, k}$, and $L>0$ is any positive integer. Let $\alpha: B \longrightarrow A_{n}$ be any homomorphism. Let $F \subset A_{n}$ be any finite set and $\varepsilon<\varepsilon_{1}$ be any positive number. It follows that there are $A_{m}$ and mutually orthogonal projections $P, Q \in A_{m}$ with $\phi_{n, m}\left(\mathbf{1}_{A_{n}}\right)=P+Q$, a unital map $\theta \in \operatorname{Map}\left(A_{n}, P A_{m} P\right)_{1}$, and a unital homomorphism $\xi \in \operatorname{Hom}\left(A_{n}, Q A_{m} Q\right)_{1}$ such that

(1) $\left\|\phi_{n, m}(f)-(\theta(f) \oplus \xi(f))\right\|<\varepsilon$, for all $f \in F$,

(2) there is a homomorphism $\alpha_{1}: B \longrightarrow P A_{m} P$ such that

$$
\begin{gathered}
\left\|\alpha_{1}^{i, j}(g)-(\theta \circ \alpha)^{i, j}(g)\right\|<3 \varepsilon_{1} \quad \forall g \in \widetilde{G}^{i}, \quad \text { if } B^{i} \quad \text { is of form } \quad M_{\bullet}\left(C\left(T_{I I, k}\right)\right), \\
\left\|\alpha_{1}^{i, j}(g)-(\theta \circ \alpha)^{i, j}(g)\right\|<\varepsilon \quad \forall g \in G^{i}, \quad \text { if } B^{i} \quad \text { is not of form } \quad M_{\bullet}\left(C\left(T_{I I, k}\right)\right) .
\end{gathered}
$$

(3) $\omega(\theta(F))<\varepsilon$ and $\theta$ is $F-\varepsilon$ multiplicative.

(4) $\xi$ factors through a $C^{*}$-algebra $C$-a direct sum of matrix algebras over $C[0,1]$ or $\mathbb{C}$ as

$$
\xi: A_{n} \stackrel{\xi_{1}}{\rightarrow} C \stackrel{\xi_{2}}{\rightarrow} Q A_{m} Q
$$

The proof is similar to Proposition 2.8 and is omitted.

2.10. Let $\alpha: \mathbb{Z} \longrightarrow \mathbb{Z} / k_{1} \mathbb{Z}$ be the group homomorphism defined by $\alpha(\mathbf{1})=[\mathbf{1}]$, where the right hand side is the equivalent class $[\mathbf{1}]$ of $\mathbf{1}$ in $\mathbb{Z} / k_{1} \mathbb{Z}$. Then it is well known from homological algebra that for the group $\mathbb{Z} / k \mathbb{Z}, \alpha$ induces a surjective map

$$
\alpha_{*}: \operatorname{Ext}(\mathbb{Z} / k \mathbb{Z}, \mathbb{Z})(=\mathbb{Z} / k \mathbb{Z}) \longrightarrow \operatorname{Ext}\left(\mathbb{Z} / k \mathbb{Z}, \mathbb{Z} / k_{1} \mathbb{Z}\right)\left(=\mathbb{Z} /\left(k, k_{1}\right) \mathbb{Z}\right),
$$


where $\left(k, k_{1}\right)$ is the greatest common factor of $k$ and $k_{1}$.

Recall, as in [DN], for two connected finite simplicial complexes $X$ and $Y$, we use $k k(Y, X)$ to denote the group of equivalent classes of homomorphisms from $C_{0}(X \backslash\{p t\})$ to $C_{0}(Y \backslash\{p t\}) \otimes$ $\mathcal{K}(H)$. Please see $[\mathrm{DN}]$ for details.

Lemma 2.11. (a) Any unital homomorphism

$$
\phi: C\left(T_{I I, k}\right) \longrightarrow M_{\bullet}\left(C\left(T_{I I I, k_{1}}\right)\right),
$$

is homotopy equivalent to unital homomorphism $\psi$ factor as

$$
C\left(T_{I I, k}\right) \stackrel{\psi_{1}}{\longrightarrow} C\left(S^{1}\right) \stackrel{\psi_{2}}{\longrightarrow} M_{\bullet}\left(C\left(T_{I I I, k_{1}}\right)\right) .
$$

(b) Any unital homomorphism $\phi: C\left(T_{I I, k}\right) \longrightarrow P M_{\bullet}\left(C\left(S^{2}\right)\right) P$ is homotopy equivalent to unital homomorphism $\psi$ factor as

$$
C\left(T_{I I, k}\right) \stackrel{\psi_{1}}{\longrightarrow} \mathbb{C} \stackrel{\psi_{2}}{\longrightarrow} P M_{\bullet}\left(C\left(S^{2}\right)\right) P .
$$

Proof. Part (b) is well known (see chapter 3 of [EG2]). To prove part (a), we note that

$$
K K\left(C_{0}\left(S^{1} \backslash\{1\}\right), C_{0}\left(T_{I I I, k_{1}} \backslash\left\{x_{1}\right\}\right)=k k\left(T_{I I I, k_{1}}, S^{1}\right)=\mathbb{Z} / k_{1} \mathbb{Z}=\operatorname{Hom}\left(\mathbb{Z}, \mathbb{Z} / k_{1} \mathbb{Z}\right)\right),
$$

where $x_{1} \in T_{I I I, k_{1}}$ is a base point. The map $\alpha: \mathbb{Z} \longrightarrow \mathbb{Z} / k_{1} \mathbb{Z}$ in 2.10 can be induced by a homomorphism: $\psi_{2}: C\left(S^{1}\right) \longrightarrow M_{\bullet}\left(C\left(T_{I I I, k}\right)\right)$.

Let

$$
[\phi] \in k k\left(T_{I I I, k_{1}} T_{I I, k}\right)=\operatorname{Ext}\left(K_{0}\left(C_{0}\left(T_{I I, k} \backslash\left\{x_{0}\right\}\right)\right), K_{1}\left(C_{0}\left(T_{I I I, k}\right)\right)\right),
$$

be the element induced by homomorphism $\phi$, where $\left\{x_{0}\right\}$ is the base point. By 2.10

$$
[\phi]=\beta \times\left[\psi_{2}\right], \text { for } \beta \in k k\left(S^{1}, T_{I I, k}\right)=\operatorname{Ext}\left(K_{0}\left(C\left(T_{I I, k} \backslash\left\{x_{0}\right\}\right)\right), K_{1}\left(C\left(S^{1}\right)\right)\right) \text {, }
$$

on the other hand $\beta$ can be realized by unital homomorphism

$$
\psi_{1}: C\left(T_{I I, k}\right) \longrightarrow C\left(S^{1}\right) .
$$

(see section 3 of [EG2]).

The following result is modification of Theorem 3.12 of [GJLP2].

Theorem 2.12. Let $B_{1}=\bigoplus_{i=1}^{s} B_{1}^{i}$, each $B^{i}$ is either matrix algebras over $\{p t\},[0,1], S^{1}$ or $\left\{T_{I I, k}\right\}_{k=2}^{\infty}$ or a dimension drop algebra. Let $\varepsilon_{1}>0$ and let

$$
\widetilde{G}_{1}\left(=\oplus \widetilde{G}_{1}^{i}\right) \subset G_{1}\left(=\oplus G_{1}^{i}\right) \subset B_{1}\left(=\oplus B_{1}^{i}\right),
$$

be a finite set with $\omega\left(G_{1}^{i}\right)<\varepsilon_{1}$ for $B_{1}^{i}=M_{\bullet}\left(C\left(T_{I I, k}\right)\right)$. 
Let $A=M_{N}(C(X))$, where $X$ is one of form $\{p t\},[0,1], S^{1},\left\{T_{I I, k}\right\}_{k=2}^{\infty},\left\{T_{I I I, k}\right\}_{k=2}^{\infty}$ or $S^{2}$. Let $\alpha_{1}: B_{1} \longrightarrow A$ be a homomorphism. Let $F_{1} \subset A$ be a finite set, and let $\varepsilon\left(<\varepsilon_{1}\right)$ and $\delta$ be any positive number. Then there exists a diagram.

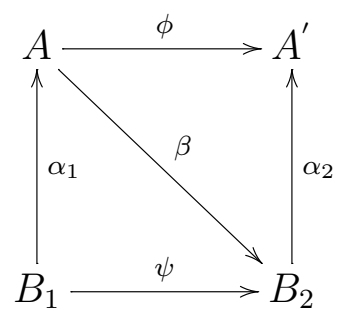

where $A^{\prime}=M_{K}(A), B_{2}$ is described as below. $B_{2}$ is a direct sum of finite dimensional $C^{*}$-algebra and dimension drop algebra if $X=T_{I I I, k} . B_{2}$ is a finite dimensional algebra if $X=S^{2}$. And $B_{2}=M_{\bullet}(A)$ if $X=\{p t\},[0,1], S^{1}, T_{I I, k}$. Furthermore the diagram satisfies the following conditions

(1) $\psi$ is a homomorphism, $\alpha_{2}$ is a unital injective homomorphism and $\phi$ is a unital simple embedding;

(2) $\beta \in \operatorname{Map}\left(A, B_{2}\right)_{1}$ is $F_{1}-\delta$ multiplicative;

(3) if $B_{1}^{i}$ is of form $M_{\bullet}\left(C\left(T_{I I, k}\right)\right)$, then

$$
\left\|\psi(g)-\beta \circ \alpha_{1}(g)\right\|<10 \varepsilon_{1}, \quad \forall g \in \widetilde{G}_{1}^{i} ;
$$

and if $B_{1}^{i}$ is not of form $M_{\bullet}\left(C\left(T_{I I, k}\right)\right)$, then

$$
\left\|\psi(g)-\beta \circ \alpha_{1}(g)\right\|<\varepsilon, \quad \forall g \in G_{1}^{i} ;
$$

(4) If $X=T_{I I, k}$, then $\omega\left(\beta\left(F_{1}\right) \cup \psi\left(G_{1}\right)\right)<\varepsilon$.

(Note that, we only required that the weak variation of finite set in $M_{\bullet}\left(C\left(T_{I I, k}\right)\right.$ to be small. In particular, we do not need to introduce the concept of weak variation for a finite subset of a dimension drop algebra.)

Proof. . For $X=T_{I I, k},\{p t\},[0,1]$ or $S^{1}$, one can choose $B_{2}=M_{K}(A)=A^{\prime}$ and homomorphism $\phi=\beta: A \longrightarrow B_{2}$ being simple embedding such that

$$
\left.\omega\left(\beta\left(F_{1}\right) \cup \alpha_{1}\left(G_{1}\right)\right)\right)<\varepsilon .
$$

This can be done by choosing $K$ large enough. Choose $\psi=\beta \circ \alpha_{1}$, and $\alpha_{2}=i d: B_{2} \longrightarrow A^{\prime}$. For the case $X=T_{I I I, k}$, or $S^{2}$, the requirement (4) is an empty requirement.

We will deal with each block of $B_{1}$ separately. For the block $B_{1}^{i}$ other than $M_{\bullet}\left(C\left(T_{I I, k}\right)\right.$, the construction can be done easily by using Lemma 2.7 , since $B_{1}^{i}$ is stably generated, which implies that any sufficiently multiplicative map from $B_{1}^{i}$ is close to a homomorphism. So we assume that $B_{1}^{i}=M_{\bullet}\left(C\left(T_{I I, k}\right)\right.$. Recall we already assume $A$ is of form $M_{\bullet}\left(C\left(T_{I I I, k}\right)\right)$ or $M_{\bullet}\left(C\left(S^{2}\right)\right.$. By Lemma 2.11, the homomorphism $\alpha_{1}: B_{1}^{i} \longrightarrow A$ is a homotopy to $\alpha^{\prime}: B_{1}^{i} \longrightarrow$ 
$A$ with $\alpha^{\prime}\left(\mathbf{1}_{B_{1}^{i}}\right)=\alpha_{1}\left(\mathbf{1}_{B_{1}^{i}}\right)$ and $\alpha^{\prime}$ factor as

$$
B_{1}^{i} \stackrel{\xi_{1}}{\rightarrow} C \stackrel{\xi_{2}}{\rightarrow} A
$$

where $C$ is a finite dimensional $C^{*}$-algebra for the case $X=S^{2}$ or $C=M_{\bullet}\left(C\left(S^{1}\right)\right.$ for the case $X=T_{I I I, k}$ (note that $B_{1}^{i}=M_{\bullet}\left(C\left(T_{I I, k}\right)\right)$. Since $C$ is stably generated, there is a finite set $E_{1} \subset A$ and $\delta_{1}>0$ such that if a complete positive map $\beta: A \longrightarrow D$ (for any $C^{*}$-algebra $D$ ) is $E_{1}-\delta_{1}$ multiplicative, then the map $\beta \circ \xi_{2}: C \longrightarrow D$ can be perturbed to a homomorphism

$$
\widetilde{\xi}: C \longrightarrow D
$$

such that

$$
\left\|\widetilde{\xi}(g)-\beta \circ \xi_{2}(g)\right\|<\varepsilon_{1}, \forall g \in \xi_{1}\left(\widetilde{G}_{1}^{i}\right) .
$$

Apply Theorem 1.6.9 of [G5] to two homotopic homomorphism

$$
\alpha_{1}, \alpha^{\prime}: B_{1}^{i} \longrightarrow A
$$

and $G_{1}^{i} \subset B_{1}^{i}$ which is approximately constant to within $\varepsilon_{1}$, to obtain a finite set $E_{2} \subset A$, $\delta_{2}>0$ and positive integer $L^{\prime}>0$ (in places of $G, \delta$ and $L$ in Theorem 1.6.9 of [G5]). Apply Lemma 2.7 to the set $\widetilde{E}=E_{1} \cup E_{2} \cup F_{1}$ and $\widetilde{\delta}=\frac{1}{3} \min \left(\varepsilon, \delta, \delta_{1}, \delta_{2}\right)$ to obtain the diagram



with $\beta^{\prime}$ being $\widetilde{E}-\widetilde{\delta}$ multiplicative and

$$
\left\|\iota \circ \beta^{\prime}(f)-\phi^{\prime}(f)\right\|<\widetilde{\delta}, \forall f \in \widetilde{E} .
$$

Let $L=L^{\prime} \cdot \operatorname{rank}\left(\mathbf{1}_{A}\right)$, and let $\beta_{1}: A \longrightarrow M_{L}\left(B^{\prime}\right)$ be any unital homomorphism defined by point evaluation. Then by Theorem 1.6 .9 of [G5], there is a unitary $u \in M_{L+1}(B)$ such that

$$
\left\|u\left(\left(\beta^{\prime} \oplus \beta_{1}\right) \circ \alpha^{\prime}(f)\right) u^{*}-\left(\beta^{\prime} \oplus \beta_{1}\right) \circ \alpha_{1}(f)\right\|<8 \varepsilon_{1}, \forall f \in \widetilde{G}_{1}^{i} .
$$

By the choice of $E_{1}$, there is a homomorphism

$$
\widetilde{\xi}: C \longrightarrow M_{L+1}\left(B^{\prime}\right),
$$

such that

$$
\left\|\widetilde{\xi}(f)-u\left(\left(\beta^{\prime} \oplus \beta\right) \circ \xi_{2}(f)\right) u^{*}\right\|<\varepsilon_{1}, \forall f \in \xi_{1}\left(\widetilde{G}_{1}^{i}\right) .
$$

Define $B_{2}=M_{L+1}\left(B^{\prime}\right), K=L_{1}(L+1), A^{\prime}=M_{K}(A)=M_{L+1}\left(M_{L_{1}}(A)\right)$, 


$$
\begin{gathered}
\psi: B_{1}^{i} \longrightarrow B_{2} \text { by } \psi=\widetilde{\xi} \circ \xi_{1}: B_{1}^{i} \stackrel{\xi_{1}}{\longrightarrow} C \stackrel{\widetilde{\xi}}{\rightarrow} B_{2}, \\
\beta: A \longrightarrow M_{L+1}\left(B^{\prime}\right) \text { by } \beta=\beta^{\prime} \oplus \beta_{1},
\end{gathered}
$$

and

$$
\phi: A \longrightarrow M_{L+1}\left(M_{L_{1}}(A)\right) \text { by } \phi=\phi^{\prime} \oplus\left(\left(\iota \otimes i d_{L}\right) \circ \beta_{1}\right)
$$

(note that $\beta_{1}$ is a homomorphism) to finish the proof.

2.13. Recall for $A=\bigoplus_{i=1}^{t} M_{k_{i}}\left(C\left(X_{i}\right)\right)$, where $X_{i}$ are path connected simplicial complexs, we use the notation $r(A)$ to denote $\bigoplus_{i=1}^{t} M_{k_{i}}(\mathbb{C})$ which could be considered to be the subalgebra consisting of all t-tuples of constant function from $X_{i}$ to $M_{k_{i}}(\mathbb{C})(i=1,2, \cdots, t)$. Fixed a base point $x_{i}^{0} \in X_{i}$ for each $X_{i}$, one defines a map $r: A \longrightarrow r(A)$ by

$$
r\left(f_{1}, f_{2}, \cdots, f_{t}\right)=\left(f_{1}\left(x_{1}^{0}\right), f_{2}\left(x_{2}^{0}\right), \cdots, f_{t}\left(x_{t}^{0}\right)\right) \in r(A) .
$$

We have the following Corollary

Corollary 2.14. Let $B_{1}=\oplus B_{1}^{j}$, where $B_{1}^{j}$ are either of form $M_{k(j)}\left(C\left(X_{j}\right)\right)$, with $X_{j}$ being one of $\{p t\},[0,1], S^{1},\left\{T_{I I, k}\right\}_{k=2}^{\infty}$ or one of form $M_{k(j)}\left(I_{l(j)}\right)$. Let $\alpha_{1} ; B_{1} \longrightarrow A$ be a homomorphism, where $A$ is a direct sum of matrix algebras over $\{p t\},[0,1], S^{1},\left\{T_{I I, k}\right\}_{k=2}^{\infty},\left\{T_{I I I, k}\right\}_{k=2}^{\infty}$ and $S^{2}$. Let $\varepsilon_{1}>0$ and let $\widetilde{E}\left(=\oplus \widetilde{E}^{i}\right) \subset E\left(=\oplus E^{i}\right) \subset B_{1}\left(=\oplus B_{1}^{i}\right)$ be two finite subset with the condition

$$
\omega\left(\widetilde{E}^{i}\right)<\varepsilon_{1} \text {, if } B_{1}^{i}=M_{\bullet}\left(C\left(Y_{i}\right)\right) \text { with } Y_{i} \in\left\{T_{I I, k}\right\}_{k=2}^{\infty} .
$$

Let $F \subset A$ be any finite set, $\varepsilon_{2}>0, \delta>0$. Then there exists a diagram

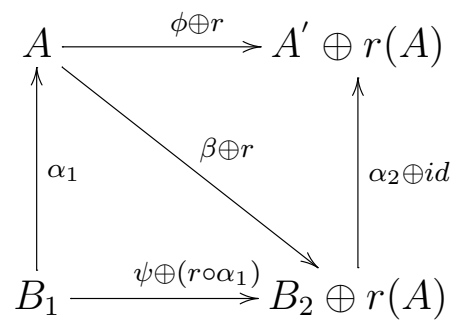

where $A^{\prime}=M_{L}(A)$, and $B_{2}$ is a direct sum of matrix algebras over space: $\{p t\},[0,1], S^{1}$, $\left\{T_{I I, k}\right\}_{k=2}^{\infty}$ and dimension drop algebras, with the following properties.

(1) $\psi$ is a homomorphism, $\alpha_{2}$ is a injective homomorphism and $\phi$ is a unital simple embedding. 
(2) $\beta \in \operatorname{Map}\left(A, B_{2}\right)_{1}$ is $F_{1}-\delta$ multiplicative.

(3) for $g \in \widetilde{E}^{i}$ with $B_{1}^{i}=M_{\bullet}\left(C\left(X_{i}\right)\right), X_{i} \in\left\{T_{I I, k}\right\}_{k=2}^{\infty}$ we have

$$
\left\|(\beta \oplus r)(g)-\left(\psi \oplus\left(r \circ \alpha_{1}\right)\right)(g)\right\| \leqslant 10 \varepsilon_{1} ;
$$

and for $g \in E^{i}\left(\supset \widetilde{E}^{i}\right)$ where $B_{1}^{i}$ is not of form $M_{\bullet}\left(C\left(T_{I I, k}\right)\right)$, we have

$$
\left\|(\beta \oplus r)(g)-\left(\psi \oplus\left(r \circ \alpha_{1}\right)\right)(g)\right\|<\varepsilon_{1} ;
$$

and for $f \in F$

$$
\left\|\left(\alpha_{2} \oplus i d\right) \circ(\beta \oplus r)(f)-(\phi \oplus r)(f)\right\|<\varepsilon_{1} .
$$

(4) For $B_{2}^{i}$ of form $M_{\bullet}\left(C\left(T_{I I, k}\right)\right)$,

$$
\omega\left(\pi_{i}(\beta(F) \cup \psi(E))\right)<\varepsilon_{2},
$$

where $\pi_{i}$ is the canonical projection from $B_{2}$ to $B_{2}^{i}$.

Remark: In the application of the above Corollary, we will denote the map $\beta \oplus r$ by $\beta$ and $\psi \oplus\left(r \circ \alpha_{1}\right)$ by $\psi$.

\section{§3. The proof of main theorem}

In this section, we prove the following main theorem.

Theorem 3.1. Suppose $\lim \left(A_{n}=\bigoplus_{i=1}^{t_{n}} M_{[n, i]}\left(C\left(X_{n, i}\right)\right), \phi_{n, m}\right)$ is an $A H$ inductive limit with $X_{n, i}$ being among the spaces $\{p t\},[0,1], S^{1},\left\{T_{I I, k}\right\}_{k=2}^{\infty},\left\{T_{I I I, k}\right\}_{k=2}^{\infty}$, such that the limit algebra $A$ has ideal property. Then there is another inductive system $\left(B_{n}=\oplus B_{n}^{i}, \psi_{n, m}\right)$ with same limit algebra, where $B_{n}^{i}$ are either $M_{[n, i]^{\prime}}\left(C\left(Y_{n, i}\right)\right)$ with $Y_{n, i}$ being one of $\{p t\},[0,1], S^{1},\left\{T_{I I, k}\right\}_{k=2}^{\infty}$ (but without $T_{I I I, k}$ and $S^{2}$ ), or dimension drop algebra $M_{[n, i]^{\prime}}\left(I_{k(n, i)}\right)$.

Proof. Let $\varepsilon_{1}>\varepsilon_{2}>\varepsilon_{3}>\cdots$ be a sequence of positive number with $\sum \varepsilon_{n}<+\infty$. We need to construct the intertwining diagram 


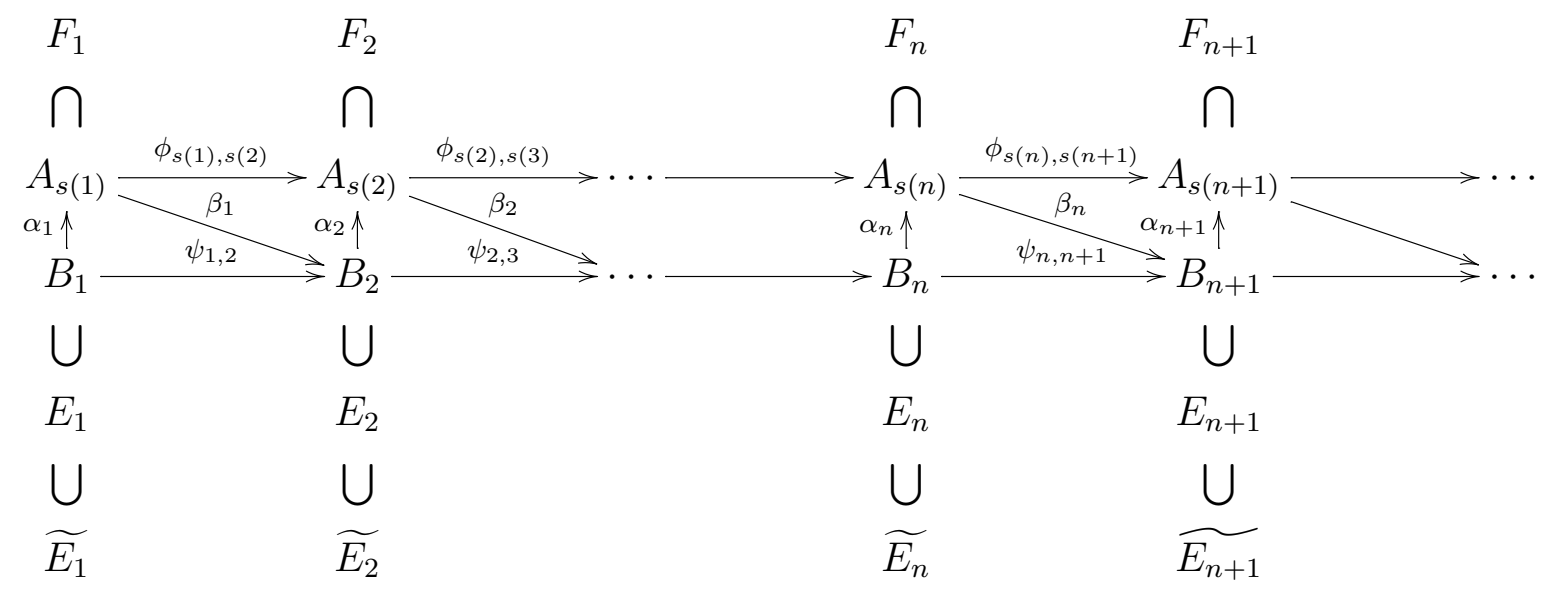

satisfying the following conditions

(0.1) $\left(A_{s(n)}, \phi_{s(n), s(m)}\right)$ is a sub-inductive system of $\left(A_{n}, \phi_{n, m}\right),\left(B_{n}, \psi_{n, m}\right)$ is an inductive system of direct sum of matrix algebras over the spaces $\{p t\},[0,1], S^{1}, T_{I I, k}$ and dimension drop algebra $M_{\bullet}\left(I_{k(n, i)}\right)$.

(0.2) Choose $\left\{a_{i, j}\right\}_{j=1}^{\infty} \subset A_{s(i)}$ and $\left\{b_{i, j}\right\}_{j=1}^{\infty} \subset B_{i}$ to be countable dense subsets of unit balls of $A_{s(i)}$ and $B_{i}$, respectively. $F_{n}$ are subsets of unit balls of $A_{s(n)}$, and $\widetilde{E_{n}} \subset E_{n}$ are both subsets of unit balls of $B_{n}$ satisfying

$$
\begin{gathered}
\phi_{s(n), s(n+1)}\left(F_{n}\right) \cup \alpha_{n+1}\left(E_{n+1}\right) \cup \bigcup_{i=1}^{n+1} \phi_{s(i), s(n+1)}\left(\left\{a_{i 1}, a_{i 2}, \cdots, a_{i n+1}\right\}\right) \subset F_{n+1}, \\
\psi_{n, n+1}\left(E_{n}\right) \cup \beta_{n}\left(F_{n}\right) \subset \widetilde{E}_{n+1} \subset E_{n+1},
\end{gathered}
$$

and

$$
\bigcup_{i=1}^{n+1} \psi_{i, n+1}\left(\left\{b_{i 1}, b_{i 2}, \cdots, b_{i n+1}\right\}\right) \subset E_{n+1} .
$$

(Here $\phi_{n, n}: A_{n} \longrightarrow A_{n}$, and $\psi_{n, n}: B_{n} \longrightarrow B_{n}$ are understand as identity maps.)

(0.3) $\beta_{n}$ are $F_{n}-2 \varepsilon_{n}$ multiplicative and $\alpha_{n}$ are homomorphism.

(0.4) for all $g \in \widetilde{E}_{n}$,

$$
\left\|\psi_{n, n+1}(g)-\beta_{n} \circ \alpha_{n}(g)\right\|<14 \varepsilon_{n},
$$

and for all $f \in F_{n}$,

$$
\left\|\phi_{s(n), s(n+1)}(f)-\alpha_{n+1} \circ \beta_{n}(f)\right\|<14 \varepsilon_{n} .
$$

(0.5) For any block $B_{n}^{i}$ with spectrum $T_{I I, k}$, we have $\omega\left(\widetilde{E}_{n}^{i}\right)<\varepsilon_{n}$, where $\widetilde{E}_{n}^{i}=\pi_{i}\left(\widetilde{E}_{n}\right)$ for $\pi_{i}: B_{n} \longrightarrow B_{n}^{i}$ the canonical projections.

The diagram will be constructed inductively. First, let $B_{1}=\{0\}, A_{s(1)}=A_{1}, \alpha_{1}=0$. Let $b_{1 j}=0 \in B_{1}$ for $j=1,2, \ldots$ and let $\left\{a_{1 j}\right\}_{j=1}^{\infty}$ be a countable dense subset of the unit ball of $A_{s(1)}$. And let $\widetilde{E}_{1}=E_{1}=\left\{b_{11}\right\}=B_{1}$ and $F_{1}=\bigoplus_{i=1}^{t_{1}} F_{1}^{i}$, where $F_{1}^{i}=\pi_{i}\left(\left\{a_{11}\right\}\right) \subset A_{1}^{i}$. 
As inductive assumption, assume that we already have the diagram

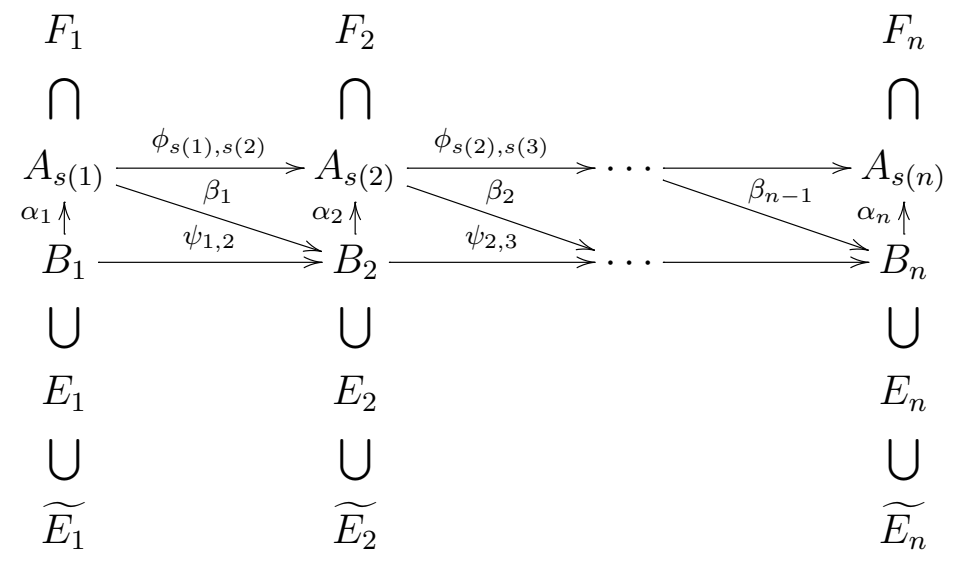

and for each $i=1,2, \cdots, n$, we have dense subsets $\left\{a_{i j}\right\}_{j=1}^{\infty} \subset$ the unit ball of $A_{s(i)}$ and $\left\{b_{i j}\right\}_{j=1}^{\infty} \subset$ the unit ball of $B_{i}$ satisfying the conditions (0.1)-(0.5) above. We have to construct the next piece of the diagram

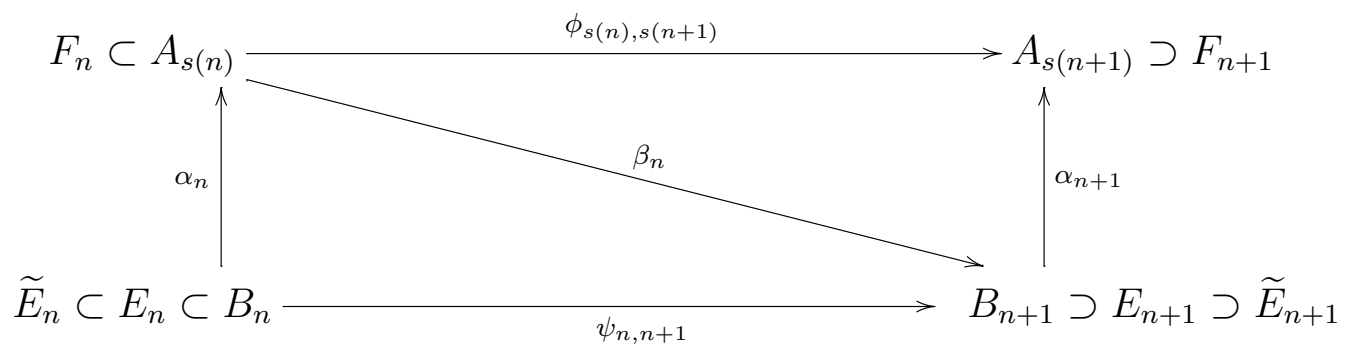

to satisfy the condition (0.1)-(0.5).

Among the conditions for induction assumption, we will only use the conditions that $\alpha_{n}$ is a homomorphism and (0.5) above.

Step 1. We enlarge $\widetilde{E}_{n}$ to $\bigoplus_{i} \pi_{i}\left(\widetilde{E}_{n}^{i}\right)$ and $E_{n}$ to $\bigoplus_{i} \pi_{i}\left(E_{n}\right)$. Then $\widetilde{E}_{n}\left(=\oplus \widetilde{E}_{n}^{i}\right) \subset E_{n}\left(=\oplus E_{n}\right)$ and for each $B_{n}^{i}$ with spectrum $T_{I I, k}$, we have $\omega\left(E_{n}^{i}\right)<\varepsilon_{n}$ from induction assumption (0.5). By proposition 2.9 applied to $\alpha_{n}: B_{n} \rightarrow A_{s(n)}, \widetilde{E}_{n} \subset E_{n} \subset B_{n}, F_{n} \subset A_{s(n)}$ and $\varepsilon_{n}>0$, there are $A_{m_{1}}\left(m_{1}>s(n)\right)$, two othogonal projections $P_{0}, P_{1} \in A_{m_{1}}$ with $\phi_{s(n), m_{1}}\left(\mathbf{1}_{A_{s(n)}}\right)=P_{0}+P_{1}$ and $P_{0}$ trivial, a $C^{*}$-algebra $C$-a direct sum of matrix algebras over $C[0,1]$ or $\mathbb{C}$, a unital map $\theta \in \operatorname{Map}\left(A_{s(n)}, P_{0} A_{m_{1}} P_{0}\right)_{1}$, a unital homomorphism $\xi_{1} \in \operatorname{Hom}\left(A_{s(n)}, C\right)_{1}$, a unital homomorphism $\xi_{2} \in \operatorname{Hom}\left(C, P_{1} A_{m_{1}} P_{1}\right)_{1}$ such that

(1.1) $\left\|\phi_{s(n), m_{1}}(f)-\theta(f) \oplus\left(\xi_{2} \circ \xi_{1}\right)(f)\right\|<\varepsilon_{n}$ for all $f \in F_{n}$.

(1.2) $\theta$ is $F_{n}-\varepsilon$ multiplicative and $F:=\theta\left(F_{n}\right)$ satisfy $\omega(F)<\varepsilon_{n}$.

(1.3) $\left\|\alpha(g)-\theta \circ \alpha_{n}(g)\right\|<3 \varepsilon_{n}$ for all $g \in \widetilde{E}_{n}$.

Let all the blocks of $\mathrm{C}$ be parts of $C^{*}$-algebra $B_{n+1}$. That is 


$$
B_{n+1}=C \oplus \text { (some other blocks). }
$$

The map $\beta_{n}: A_{s(n)} \rightarrow B_{n+1}$, and the homomorphism $\psi_{n, n+1}: B_{n} \rightarrow B_{n+1}$ are defined by $\beta_{n}=\xi_{1}: A_{s(n)} \rightarrow C\left(\subset B_{n+1}\right)$ and $\psi_{n, n+1}=\xi_{1} \circ \alpha_{n}: B_{n} \rightarrow C\left(\subset B_{n+1}\right)$ for the blocks of $C\left(\subset B_{n+1}\right)$. For this part, $\beta_{n}$ is also a homomorphism.

Step 2. Let $A=P_{0} A_{m_{1}} P_{0}, F=\theta\left(F_{n}\right)$. Since $P_{0}$ is a trivial projection,

$$
A \cong \oplus M_{l_{i}}\left(C\left(X_{m_{1}, i}\right)\right) .
$$

Let $r(A):=\oplus M_{l_{i}}(\mathbb{C})$ and $r: A \rightarrow r(A)$ be as in 2.13. Applying corollary 2.14 and its remark to $\alpha: B_{n} \rightarrow A, \widetilde{E}_{n} \subset E_{n} \subset B_{n}$ and $F \subset A$, we obtain the following diagram

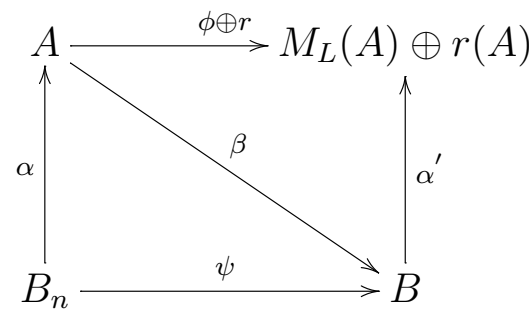

such that

(2.1) $B$ is a direct sum of matrix algebras over $\{p t\},[0,1], S^{1}, T_{I I, k}$ and dimension drop algebra.

(2.2) $\alpha^{\prime}$ is an injective homomorphism and $\beta$ is $F-\varepsilon_{n}$ multiplicative.

(2.3) $\phi: A \rightarrow M_{L}(A)$ is a unital simple embedding and $r: A \rightarrow r(A)$ is as in 2.13.

(2.4) $\|\beta \circ \alpha(g)-\psi(g)\|<10 \varepsilon_{n}$ for all $g \in \widetilde{E}_{n}$ and $\left\|(\phi \oplus r)(f)-\alpha^{\prime} \circ \beta(f)\right\|<\varepsilon_{n}$ for all $f \in F\left(:=\theta\left(F_{n}\right)\right)$.

(2.5) $\omega\left(\pi_{i}\left(\psi\left(E_{n}\right)\right) \cup \beta(F)\right)<\varepsilon_{n+1}$ (note that $\beta(F)=\beta \circ \theta\left(F_{n}\right)$ ), for $B_{n}^{i}$ being of form $M_{\bullet}(C(X))$ with $X \in\left\{T_{I I, k}\right\}_{k=2}^{\infty}$.

Let all the blocks $B$ be also part of $B_{n+1}$, that is

$$
B_{n+1}=C \oplus B \oplus \text { (some other blocks). }
$$

The maps $\beta_{n}: A_{s(n)} \longrightarrow B_{n+1}, \psi_{n, n+1}: B_{n} \longrightarrow B_{n+1}$ are defined by

$$
\beta_{n}:=\beta \circ \theta: A_{s(n)} \stackrel{\theta}{\rightarrow} A \stackrel{\beta}{\rightarrow} B\left(\subset B_{n+1}\right),
$$

and

$$
\psi_{n, n+1}:=\psi: B_{n} \rightarrow B\left(\subset B_{n+1}\right),
$$

for the blocks of $B\left(\subset B_{n+1}\right)$. This part of $\beta_{n}$ is $F_{n}-2 \varepsilon_{n}$ multiplicative, since $\theta$ is $F_{n}-\varepsilon_{n}$ multiplicative, $\beta$ is $F-\varepsilon_{n}$ multiplicative and $F=\theta\left(F_{n}\right)$.

Step 3. By Lemma 3.15 of [GJLP2] applied to $\phi \oplus r: A \rightarrow M_{L}(A) \oplus r(A)$, there is an $A_{m_{2}}$ and there is a unital homomorphism

$$
\lambda: M_{L}(A) \oplus r(A) \rightarrow R A_{m_{2}} R,
$$


where $R=\phi_{m_{1}, m_{2}}\left(P_{0}\right)$ (write $R$ as $\bigoplus_{j} R^{j} \in \bigoplus_{j} A_{m}^{j}$ ) such that the diagram

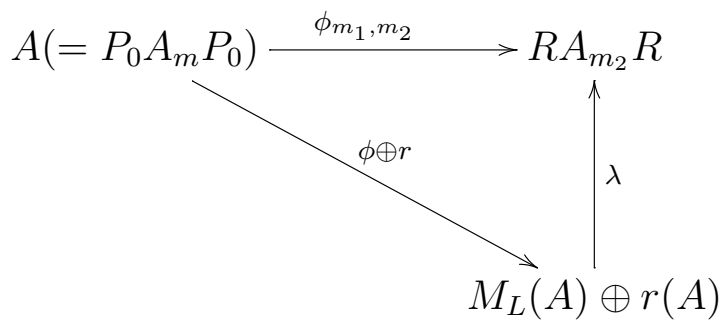

satisfies the following conditions:

(3.1) $\lambda \circ(\phi \oplus r)$ is homotopy equivalent to

$$
\phi^{\prime}:=\left.\phi_{m_{1}, m_{2}}\right|_{A} .
$$

Step 4. Applying Theorem 1.6.9 of [G5] to finite set $F \subset A$ (with $\omega(F)<\varepsilon_{n}$ ) and to two homotopic homomorphisms $\phi^{\prime}$ and $\lambda \circ(\phi \oplus r): A \rightarrow R A_{m_{2}} R$ (with $R A_{m_{2}} R$ in place of $C$ in Theorem 1.6.9 of [G5]), we obtain a finite set $F^{\prime} \subset R A_{m_{2}} R, \delta>0$ and $L>0$ as in the Theorem.

Let $G=\oplus \pi_{i}\left(\psi\left(E_{n}\right) \cup \beta(F)\right)=\oplus G^{i}$. Then by (2.5), we have $\omega\left(G^{i}\right)<\varepsilon_{n+1}$, if $B^{i}$ is of form $M_{\bullet}\left(C\left(T_{I I, k}\right)\right)$. By Proposition 2.8 applied to $R A_{m_{2}} R$ and

$$
\lambda \circ \alpha^{\prime}: B \rightarrow R A_{m_{2}} R,
$$

finite set $G \subset B, F^{\prime} \cup\left(\left.\phi_{m_{1} m_{2}}\right|_{A}(F)\right) \in R A_{m_{2}} R, \min \left(\varepsilon_{n}, \delta\right)>0$ (in place of $\varepsilon$ ) and $L>0$, there are $A_{s(n+1)}$, mutually orthogonal projections $Q_{0}, Q_{1}, Q_{2} \in A_{s(n+1)}$ with $\phi_{m_{2}, s(n+1)}(R)=$ $Q_{0} \oplus Q_{1} \oplus Q_{2}$, a $C^{*}$-algebra $D$-a direct sum of matrix algebras over $\mathrm{C}[0,1]$ or $\mathbb{C}$ - , a unital map $\theta_{0} \in \operatorname{Map}\left(R A_{m_{2}} R, Q_{0} A_{s(n+1)} Q_{0}\right)$, and four unital homomorphisms

$$
\theta_{1} \in \operatorname{Hom}\left(R A_{m_{2}} R, Q_{1} A_{s(n+1)} Q_{1}\right)_{1}, \xi_{3} \in \operatorname{Hom}\left(R A_{m_{2}} R, D\right)_{1}, \xi_{4} \in \operatorname{Hom}\left(D, Q_{2} A_{s(n+1)} Q_{2}\right)_{1}
$$

and $\alpha^{\prime \prime} \in \operatorname{Hom}\left(B,\left(Q_{0}+Q_{1}\right) A_{s(n+1)}\left(Q_{0}+Q_{1}\right)\right)_{1}$ such that the following is true.

(4.1) $\left\|\phi_{m_{2}, s(n+1)}(f)-\left(\left(\theta_{0}+\theta_{1}\right) \oplus \xi_{4} \circ \xi_{3}\right)(f)\right\|<\varepsilon_{n}$, for all $f \in \phi_{m_{1}, m_{2}} \mid{ }_{A}(F) \subset R A_{m_{2}} R$.

(4.2) $\left\|\alpha^{\prime \prime}(g)-\left(\theta_{0}+\theta_{1}\right) \circ \lambda \circ \alpha^{\prime}(g)\right\|<3 \varepsilon_{n+1}<3 \varepsilon_{n}, \forall g \in G$.

(4.3) $\theta_{0}$ is $F^{\prime}-\min \left(\varepsilon_{n}, \delta\right)$ multiplicative and $\theta_{1}$ satisfies that

$$
\theta_{1}^{i, j}([q])>L \cdot\left[\theta_{0}^{i, j}\left(R^{i}\right)\right]
$$

for any non zero projection $q \in R^{i} A_{m_{1}} R^{i}$.

By Theorem 1.6.9 of [G5], there is a unitary $u \in\left(Q_{0} \oplus Q_{1}\right) A_{s(n+1)}\left(Q_{0}+Q_{1}\right)$ such that

$$
\left\|\left(\theta_{0}+\theta_{1}\right) \circ \phi^{\prime}(f)-A d u \circ\left(\theta_{0}+\theta_{1}\right) \circ \lambda \circ(\phi \oplus r)(f)\right\|<8 \varepsilon_{n},
$$

for all $f \in F$.

Combining with second inequality of (2.4), we have 
(4.4) $\left\|\left(\theta_{0}+\theta_{1}\right) \circ \phi^{\prime}(f)-A d u \circ\left(\theta_{0}+\theta_{1}\right) \circ \lambda \circ \alpha^{\prime} \circ \beta(f)\right\|<9 \varepsilon_{n}$ for all $f \in F$.

Step 5. Finally let all blocks of $D$ be the rest of $B_{n+1}$. Namely, let

$$
B_{n+1}=C \oplus B \oplus D,
$$

where $C$ is from Step 1, $B$ is from Step 2 and $D$ is from Step 4 .

We already have the definition of $\beta_{n}: A_{s(n)} \rightarrow B_{n+1}$ and $\psi_{n, n+1}: B_{n} \rightarrow B_{n+1}$ for those blocks of $C \oplus B \subset B_{n+1}$ (from Step 1 and Step 2). The definition of $\beta_{n}$ and $\psi_{n, n+1}$ for blocks of $D$ and the homomorphism $\alpha_{n+1}: C \oplus B \oplus D \rightarrow A_{s(n+1)}$ will be given below.

The part of $\beta_{n}: A_{s(n)} \rightarrow D\left(\subset B_{n+1}\right)$ is defined by

$$
\beta_{n}=\xi_{3} \circ \phi^{\prime} \circ \theta: A_{s(n)} \stackrel{\theta}{\longrightarrow} A \stackrel{\phi}{\longrightarrow} R A_{m_{2}} R \stackrel{\xi_{3}}{\longrightarrow} D .
$$

(Recall that $A=P_{0} A_{m_{2}} P_{0}$ and $\phi^{\prime}=\left.\phi_{m_{1}, m_{2}}\right|_{A}$.) Since $\theta$ is $F_{n}-\varepsilon_{n}$ multiplicative, and $\phi^{\prime}$ and $\xi_{3}$ are homomorphism, we know this part of $\beta_{n}$ is $F_{n}-\varepsilon_{n}$ multiplicative.

The part of $\psi_{n, n+1}: B_{n} \rightarrow D\left(\subset B_{n+1}\right)$ is defined by

$$
\psi_{n, n+1}=\xi_{3} \circ \phi^{\prime} \circ \alpha: B_{n} \stackrel{\alpha}{\longrightarrow} A \stackrel{\phi^{\prime}}{\longrightarrow} R A_{m} R \stackrel{\xi_{3}}{\longrightarrow} D
$$

which is a homomorphism.

The homomorphism $\alpha_{n+1}: C \oplus B \oplus D \rightarrow A_{s(n+1)}$ is defined as following.

Let $\phi^{\prime \prime}=\left.\phi_{m_{1}, s(n+1)}\right|_{P_{1} A_{m_{1}} P_{1}}: P_{1} A_{m_{1}} P_{1} \longrightarrow \phi_{m_{1}, s(n+1)}\left(P_{1}\right) A_{s(n+1)} \phi_{m_{1}, s(n+1)}\left(P_{1}\right)$, where $P_{1}$ is from Step 1. Define

$$
\left.\alpha_{n+1}\right|_{C}=\phi^{\prime \prime} \circ \xi_{2}: C \stackrel{\xi_{2}}{\longrightarrow} P_{1} A_{m_{1}} P_{1} \stackrel{\phi^{\prime \prime}}{\longrightarrow} \phi_{m_{1}, s(n+1)}\left(P_{1}\right) A_{s(n+1)} \phi_{m_{1}, s(n+1)}\left(P_{1}\right),
$$

where $\xi_{2}$ is from Step 1 .

$$
\left.\alpha_{n+1}\right|_{B}=A d u \circ \alpha^{\prime \prime}: B \stackrel{\alpha^{\prime \prime}}{\longrightarrow}\left(Q_{0} \oplus Q_{1}\right) A_{s(n+1)}\left(Q_{0}+Q_{1}\right) \stackrel{A d u}{\longrightarrow}\left(Q_{0} \oplus Q_{1}\right) A_{s(n+1)}\left(Q_{0}+Q_{1}\right)
$$

where $\alpha^{\prime \prime}$ is from Step 4, and define

$$
\left.\alpha_{n+1}\right|_{D}=\xi_{4}: D \rightarrow Q_{2} A_{s(n+1)} Q_{2} .
$$

Finally choose $\left\{a_{n+1, j}\right\}_{j=1}^{\infty} \subset A_{s(n+1)}$ and $\left\{b_{n+1, j}\right\}_{j=1}^{\infty} \subset B_{n+1}$ to be countable dense subsets of the unit balls of $A_{s(n+1)}$ and $B_{n+1}$, respectively. And choose

$$
F_{n+1}^{\prime}=\phi_{s(n), s(n+1)}\left(F_{n}\right) \cup \alpha_{n+1}\left(E_{n+1}\right) \cup \bigcup_{i=1}^{n+1} \phi_{s(i), s(n+1)}\left(\left\{a_{i 1}, a_{i 2}, \cdots, a_{i n+1}\right\}\right),
$$




$$
\begin{gathered}
E_{n+1}^{\prime}=\psi_{n, n+1}\left(E_{n}\right) \cup \beta_{n}\left(F_{n}\right) \cup \bigcup_{i=1}^{n+1} \psi_{i, n+1}\left(\left\{b_{i 1}, b_{i 2}, \cdots, b_{i n+1}\right\}\right), \\
\widetilde{E}_{n+1}^{\prime}=\psi_{n, n+1}\left(E_{n}\right) \cup \beta_{n}\left(F_{n}\right) \subset E_{n+1}^{\prime} .
\end{gathered}
$$

Define $F_{n+1}^{i}=\pi_{i}\left(F_{n+1}^{\prime}\right)$ and $F_{n+1}=\bigoplus_{i} F_{n+1}^{i}, E_{n+1}^{i}=\pi_{i}\left(E_{n+1}^{\prime}\right)$ and $E_{n+1}=\oplus_{i} E_{n+1}^{i}$. For those block $B_{n+1}^{i}$ inside the algebra $B$ define $\widetilde{E}_{n+1}^{i}=\pi_{i}\left(\widetilde{E}_{n+1}\right)$. For those blocks inside $C$ and $D$, define $\widetilde{E}_{n+1}^{i}=E_{n+1}^{i}$. And finally let $E_{n+1}=\bigoplus_{i} \widetilde{E}_{n+1}^{i}$. Note all the blocks with spectrum $T_{I I, k}$ are in $B$. And (2.5) tells us that for those blocks $\omega\left(\widetilde{E}_{n+1}^{i}\right)<\varepsilon_{n+1}$. Thus we obtain the following diagram



Step 6.Now we need to verify all the condition (0.1)-(0.5) for the above diagram.

From the end of Step 5, we know (0.5) holds, (0.1)-(0.2) hold from the construction (see the construction of $B, C, D$ in Step 1, 2 and 4, and $\widetilde{E}_{n+1} \subset E_{n+1}, F_{n+1}$ is the end of Step 5). (0.3) follows from the end of Step 1, the end of Step 2 and the part of definition of $\beta_{n}$ for $D$ from Step 5.

So we only need to verify $(0.4)$.

Combining (1.1) with (4.1), we have $\left\|\phi_{s(n), s(n+1)}(f)-\left[\left(\phi^{\prime \prime} \circ \xi_{2} \circ \xi_{1}\right) \oplus\left(\theta_{0}+\theta_{1}\right) \circ \phi^{\prime} \circ \theta \oplus\left(\xi_{4} \circ \xi_{3} \circ \phi^{\prime} \circ \theta\right)(f)\right](f)\right\|<\varepsilon_{n}+\varepsilon_{n}=2 \varepsilon_{n}$ for all $f \in F_{n}$ (recall that $\phi^{\prime \prime}=\left.\phi_{m_{1}, s(n+1)}\right|_{P_{1} A_{m_{1}} P_{1}}, \phi^{\prime}:=\left.\phi_{m_{1}, m_{2}}\right|_{P_{0} A_{m_{1}} P_{o}}$ ).

Combining with (4.2) and (4.4), and definition of $\beta_{n}$ and $\alpha_{n+1}$, the above inequality yields $\left\|\phi_{s(n), s(n+1)}(f)-\left(\alpha_{n+1} \circ \beta_{n+1}\right)(f)\right\|<9 \varepsilon_{n}+3 \varepsilon_{n}+2 \varepsilon_{n}=14 \varepsilon_{n}, \forall f \in F_{n}$.

Combining (1.3), the first inequality of (2.4) and the definition of $\beta_{n}$ and $\psi_{n, n+1}$, we have

$$
\left\|\psi_{n, n+1}(g)-\left(\beta_{n} \circ \alpha_{n}\right)(g)\right\|<10 \varepsilon_{n}+3 \varepsilon_{n}<14 \varepsilon_{n}, \forall g \in \widetilde{E}_{n} .
$$

So we obtain(0.4). The theorem follows from Proposition 4.1 of [GJLP2].

Note that if $q \in M_{l}\left(I_{k}\right)$, then $q M_{k}\left(I_{k}\right) q$ isomorphic to $M_{l_{1}}\left(I_{k}\right)$. Combining with the main theorem of [GJLP2] (see Theorem 4.2, and 2.7 of [GJLP2]) we have 
Theorem 3.2. Suppose that $A=\lim \left(A_{n}=\oplus P_{n, i} M_{[n, i]}\left(C\left(X_{n, i}\right)\right) P_{n, i}\right)$ is an $A H$ inductive limit with $\operatorname{dim}\left(X_{n, i}\right) \leqslant M$ for a fixed positive integer $M$ such that limit algebra $A$ has ideal property. Then $A$ can be rewrite as inductive $\operatorname{limit} \lim \left(B_{n}=\oplus B_{n}^{i}, \psi_{n, m}\right)$, where either $B_{n}^{i}=Q_{n, i} M_{[n, i]^{\prime}}\left(C\left(Y_{n, i}\right)\right) Q_{n, i}$ with $Y_{n, i}$ being one of the spaces $\{p t\},[0,1], S^{1},\left\{T_{I I, k}\right\}_{k=2}^{\infty}$ or $B_{n}^{i}=M_{[n, i]^{\prime}}\left(I_{l_{(n, i)}}\right)$ a dimension drop algebra.

\section{References}

[Blackadar] B. Blackadar, Matricial and ultra-matricial topology, Operator Algebras, Mathematical Physics, and Low Dimensional Topology (R. H. Herman and B Tanbay eds) A K Peter, Massachusetts (1993), 11-38

[D1] M. Dadarlat, Approximately unitarily equiralent, morphisms and inductive limit $C^{*}$ algebras, K-theory 9(1995), 117-137

[D2] M. Dadarlat, Reduction to dimesion these of local spectra of Real rank zero $C^{*}$-algebras, J. Reine Angew. Math. 460(1995) 189-212

[DG] M. Dadarlat and G. Gong, A classification result for approximately homogeneous $C^{*}$ algebras of real rank zero, Geometric and Functional Analysis, 7(1997) 646-711

[DN] M. Dadarlat and A. Nemethi, Sharp theory and (connective) K-theory, J. Operator Theory 23(1990) 207-291

[Ell1] G. A. Elliott, On the classification of $C^{*}$-algebras of real rank zero, J. Reine Angew. Math. 443(1993) 263-290

[Ell2] G. A. Elliott, A classification of certain simple $C^{*}$-algebras, Quantum and NonCommutative Analysis, Kluwer, Dordrecht, (1993), pp, 373-388

[Ell3] G. A. Elliott, A classification of certain simple $C^{*}$-algebras, II, J. Ramaunjan Math. Soc. 12 (1997), 97-134

[EG1] G. A. Elliott and G. Gong, On the inductive limits of matrix algebras over two-tori, American. J. Math 118(1996) 263-290

[EG2] G. A. Elliott and G. Gong, On the classification of $C^{*}$-algebras of real rank zero, II. Ann. of Math 144(1996) 497-610

[EGL1] G. A. Elliott, G. Gong and L. Li, On the classification of simple inductive limit $C^{*}$-algebras, II; The isomorphism Theorem, Invent. Math. 168(2)(2007) 249-320

[EGL2] G. A. Elliott, G. Gong and L. Li, Injectivity of the connecting maps in $A H$ inductive limit systems, Canand. Math. Bull. 26(2004) 4-10 
[G1] G. Gong, Approximation by dimension drop $C^{*}$-algebras and classification, C. R. Math. Rep. Acad. Sci Can. 16(1994)40-44

[G2] G. Gong, Classification of $C^{*}$-algebras of real rank zero and unsuspended E-equivalent types, J. Funct. Anal. 152(1998) 281-329

[G3-4] G. Gong, On inductive limit of matrix algebras over higher dimension spaces, Part I, II, Math Scand. 80(1997) 45-60, 61-100

[G5] G. Gong, On the classification of simple inductive limit $C^{*}$-algebras, I: Reduction Theorems. Doc. Math. 7(2002) 255-461

[GJL] G. Gong, C. Jiang, and L. Li, A classification of inductive limit $C^{*}$-algebras with ideal property, arXiv:1607.07581

[GJLP1] G. Gong, C. Jiang, L. Li, C. Pasnicu, AT structure of $A H$ algebras with ideal property and torsion free $K$-theory, J. Func. Anal. 58(2010) 2119-2143

[GJLP2] G. Gong, C. Jiang, L. Li, C. Pasnicu, A Reduction theorem for $A H$ algebras with ideal property, arXiv:1607.07575

[Ji-Jiang] K. Ji and C. Jiang, A complete classification of AI algebra with ideal property, Canadian. J. Math, 63(2), (2011), 381-412

[Jiang] C. Jiang, A classification of non simple $C^{*}$-algebras of tracial rank one:Inductive limit of finite direct sums of simple TAI $C^{*}$-algebras, J. Topol. Anal. 3 No.3(2011), 385-404

[Li1] L. Li, On the classification of simple $C^{*}$-algebras: Inductive limit of matrix algebras tree, Men Amer. Math, Soc. 127(605)(1997)

[Li2] L. Li, Simple inductive limit $C^{*}$-algebras: Spectra and approximation by interral algebras, J. Reine Angew Math 507(1999) 57-79

[Li3] L. Li, Classification of simple $C^{*}$-algebras: Inductive limit of matrix algebras over 1-dimensional spaces, J. Func. Anal. 192(2002) 1-51

[Li4] L. Li, Reduction to dimension two of local spectrum for simple $A H$ algebras, J. of Ramanujia Math. Soc. 21 No.4(2006) 365-390

[Pasnicu1] C. Pasnicu, On inductive limit of certain $C^{*}$-algebras of the form $C(x) \otimes F$, Trans. Amer. Math. Soc. 310(2)(1988) 703-714

[Pasnicu2] C. Pasnicu, Shape equiralence, nonstable K-theory and AH algebras, Pacific J. Math 192(2000) 159-182 